\title{
Widespread nocturnality of living birds stemming from their common ancestor
}

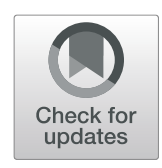

Yonghua $\mathrm{Wu}^{1,2}$ (DD

\begin{abstract}
Background: Many living birds exhibit some nocturnal activity, but the genetic basis and evolutionary origins of their nocturnality remain unknown.

Results: Here, we used a molecular phyloecological approach to analyze the adaptive evolution of 33 phototransduction genes in diverse bird lineages. Our results suggest that functional enhancement of two nightvision genes, namely, GRK1 and SLC24A1, underlies the nocturnal adaption of living birds. Further analyses showed that the diel activity patterns of birds have remained relatively unchanged since their common ancestor, suggesting that the widespread nocturnal activity of many living birds may largely stem from their common ancestor rather than independent evolution. Despite this evolutionary conservation of diel activity patterns in birds, photoresponse recovery genes were found to be frequently subjected to positive selection in diverse bird lineages, suggesting that birds generally have evolved an increased capacity for motion detection. Moreover, we detected positive selection on both dim-light vision genes and bright-light vision genes in the class Aves, suggesting divergent evolution of the vision of birds from that of reptiles and that different bird lineages have evolved certain visual adaptions to their specific light conditions.
\end{abstract}

Conclusions: This study suggests that the widespread nocturnality of extant birds has a deep evolutionary origin tracing back to their common ancestor.

Keywords: Nocturnality, Phototransduction genes, Positive selection, Diel activity pattern

\section{Background}

Living birds are generally considered diurnal, with the exception of few truly nocturnal species such as owls, nightjars and kiwis, but many diurnal birds exhibit partial or occasional nocturnal activity related to migration, dispersal, foraging, homing and singing $[1,2]$. The presence of at least some form of nocturnal activity across almost all avian lineages has long been recognized and is well documented [1,3,4]. This nocturnal activity may have been facilitated by genetic adaptations $[1,2,5]$. Indeed, a night-vision-specific brain area has been identified in nocturnally migratory songbirds [6]. While recent studies show that nocturnal taxa frequently exhibit functional enhancement of dim-light vision genes involved in

\footnotetext{
Correspondence: wuyh442@nenu.edu.cn
}

'School of Life Sciences, Northeast Normal University, 5268 Renmin Street, Changchun 130024, China

${ }^{2}$ Jilin Provincial Key Laboratory of Animal Resource Conservation and Utilization, Northeast Normal University, 2555 Jingyue Street, Changchun 130117, China the rod phototransduction pathway [7-11], it remains unknown whether this is characteristic of the evolutionary history of living birds in light of their widespread nocturnality.

It also remains unknown whether the widespread nocturnality of living birds has been retained from their common ancestors or evolved independently in diverse bird lineages. One recent study has shown that the common ancestor of living birds may have been both diurnal and nocturnal [8], and if this is true, it would suggest that the widespread nocturnality of living birds stems, in part, from their common ancestors. Alternatively, widespread nocturnality may have evolved independently in diverse bird lineages. To distinguish between these two possibilities, it is necessary to reconstruct the evolutionary history of the diel activity patterns within the class Aves.

A recently developed molecular phyloecological (MPE) approach has been demonstrated to reliably reconstruct the diel activity patterns of ancestral taxa [9]. The MPE 
approach employs phylogenetic analyses of rod and cone phototransduction genes [12, 13], which are specialized for dim-light vision and bright-light vision, respectively, and has revealed that the phylogenetic branches of nocturnal species and diurnal species are mainly characterized by positive selection on rod-expressed genes and cone-expressed genes, respectively [7, 9-11]. Following the MPE approach, we identified evidence for positive selection on two rod-expressed genes, GRK1 and $S L C 24 A 1$, and one cone-expressed gene, PDE6C, in the common ancestor branch of living birds (Fig. 1, Fig. 2), suggesting that ancestral birds were active during the day and night [8]. In this study, we extended our analyses of the same dataset to examine possible diel activity evolution within birds. Our results provide insights into the genetic bases and evolutionary origins of widespread nocturnality in birds.

\section{Results and discussion}

Using the MPE approach to infer the ancestral states of diel activity patterns [8-11], we analyzed the adaptive evolution of 33 phototransduction genes (Additional file 1) among diverse lineages of birds in the sauropsid phylogeny (Fig. 1) using the branch model, the branch-site model and clade model C implemented in PAML software [26]. Positively selected genes (PSGs) were identified using the branch-site model and clade model $\mathrm{C}$, and inferences of positive selection remained robust in terms of the initial variation in kappa and $\omega$ values.

Many internal branches of the bird clade exhibited no signals of positive selection, and only partial branches were found to be under positive selection (Fig. 1, Table 1). In Ratitae, we found one rod-expressed gene, GRK1, to be under positive selection along the common ancestor branch of the ostrich (Struthio camelus) and the emu (Dromaius novaehollandiae). GRK1 is a photoresponse recovery gene involved in the inactivation of activated rhodopsin. As photoresponse recovery is associated with motion detection [27], the positive selection on the photoresponse recovery gene suggests an increased capacity for motion detection in dim-light conditions. For Carinatae, we initially examined the positive selection along the ancestral branch (branch A in Fig. 1) and found two PSGs (GRK1 and RCVRN) (Fig. 2). Both genes are involved in photoresponse recovery, and evidence of positive selection on these genes, which was also supported by a branch-site unrestricted statistical test for episodic diversification (BUSTED), which provides a gene-wide robust test for evidence of selection (Additional file 2), suggests that ancestral Carinatae may have evolved a particularly enhanced capacity for motion detection in at least dim-light conditions. Moreover, the red-sensitive cone opsin gene LWS and the ultraviolet/ violet-sensitive cone opsin gene SWS1 were also found to be under positive selection along the Gallus gallus and Columba livia branches, respectively, suggesting an enhanced ability for bright-light vision in these lineages. One positively selected photoresponse recovery gene, $G U C Y 2 D$, was identified along the branches leading to Cuculus canorus and the common ancestor branches of Upupa epops and Picus canus, respectively. GUCY2D encodes guanylyl cyclases, which are involved in the resynthesis of cGMP, promoting photoresponse recovery. We also detected signals of positive selection on the redsensitive cone opsin gene LWS and two photoresponse recovery genes, RCVRN and GUCY2F, in two closely related groups, Passeriformes and Psittaciformes, suggesting their increased capacities for motion detection in bright-light conditions.

Among the groups examined in Carinatae, owls and falcons showed relatively strong positive selection relative to the other groups in the clade. For falcons (branch $\mathrm{H}$ in Fig. 1), we found three positively selected photoresponse recovery genes (GRK1, SLC24A1 and GUCY2D), two of which (GRK1 and SLC24A1) were rodexpressed genes (Table 1, Fig. 2). In particular, SLC24A1 encodes the $\mathrm{Na}^{+} / \mathrm{Ca}^{2+}-\mathrm{K}^{+}$ion exchanger, extruding free calcium in the outer segment of rods for the restoration of cGMP concentration. The finding of positive selection on these genes suggests that falcons have evolved an enhanced capability to detect motion in dim-light conditions, consistent with the findings of previous studies [7, 28-31]. For owls (branch G in Fig. 1), which are most active at night and during crepuscular periods, we found a marginally significant signal of positive selection on one rod-expressed gene, CNGB1 (LRT $P$-value $=0.050$ ). CNGB1 encodes the $\beta$ subunit of CNG channels and is involved in phototransduction activation. Moreover, two cone opsin genes, the red-sensitive opsin gene LWS and the blue-sensitive opsin gene $S W S 2$, showed strong signals of positive selection (LRT $P$-value $<0.001$ ) (Table 1, Fig. 2). Positive selection on these two cone opsin genes is associated with spectral tuning to maximize light abortion under crepuscular conditions [7]. When the evidence for positive selection on the PSGs found by PAML in falcons and owls was examined using BUSTED, positive selection on the gene GRK1 in falcons and the two cone opsin genes LWS and SWS2 in owls was retained (Additional file 2). In addition to the nocturnal owls, we also looked for evidence of positive selection in lineages that contain true nocturnal taxa, including Apterygiformes, Caprimulgiformes and Charadriiformes [1], for which only partial gene sequences were available, but found no evidence of positive selection in these groups. Future studies using retinal transcriptome sequencing would allow us to obtain full-length phototransduction gene sequences and perform detailed analyses of the genes underlying nightvision adaptation in these nocturnal lineages. 


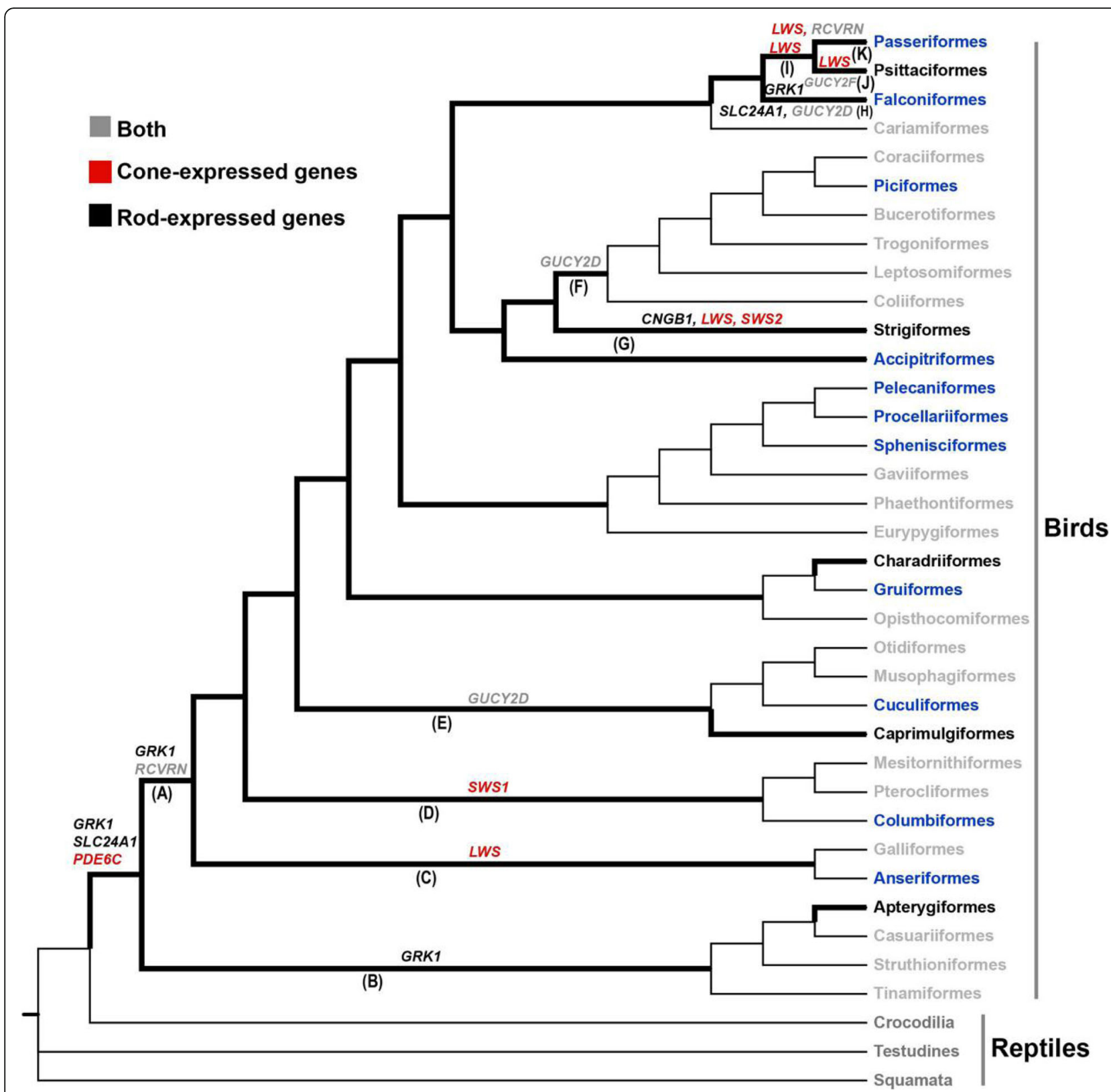

Fig. 1 Positively selected genes identified among lineages of birds. The phylogenetic relationships of bird taxa follow previous studies [14-25] and the Tree of Life Web Project (http://tolweb.org/Passeriformes). The diel activity of taxonomic bird orders follows one previous study [1]. The diurnal bird orders that contain taxa with occasional nocturnal activity are shown in blue, bird orders that harbor regular nocturnal taxa are shown in black, and diurnal bird orders with no known nocturnal activity are shown in gray. Please see Additional file 1 for species used for each of the bird orders. Bold lines indicate branches subjected to positive selection analyses in this study. The letters (A-K) in parentheses indicate the branches on which PSGs were detected in this study. The PSGs found are shown along the branches. The three PSGs found along the ancestral bird branch in our recently published study [8] are also shown. Please see the text for details about the positively selected genes identified and their corresponding specific branches

In addition to the branch model and branch-site model, we also used clade model $\mathrm{C}$ to look for evidence of positive selection on phototransduction genes in birds (Table 2). When the entire clade of birds was analyzed as a foreground clade, we detected relatively strong positive selection signals for both rod-expressed genes
(CNGA1, PDE6B, SAG and SLC24A1) and coneexpressed genes (CNGB3, PDE6C and SLC24A2), suggesting that both the dim-light vision and bright-light vision of birds were subject to divergent selection compared to those of the reptiles included in the study. This finding is likely a result of the differential adaptation of 


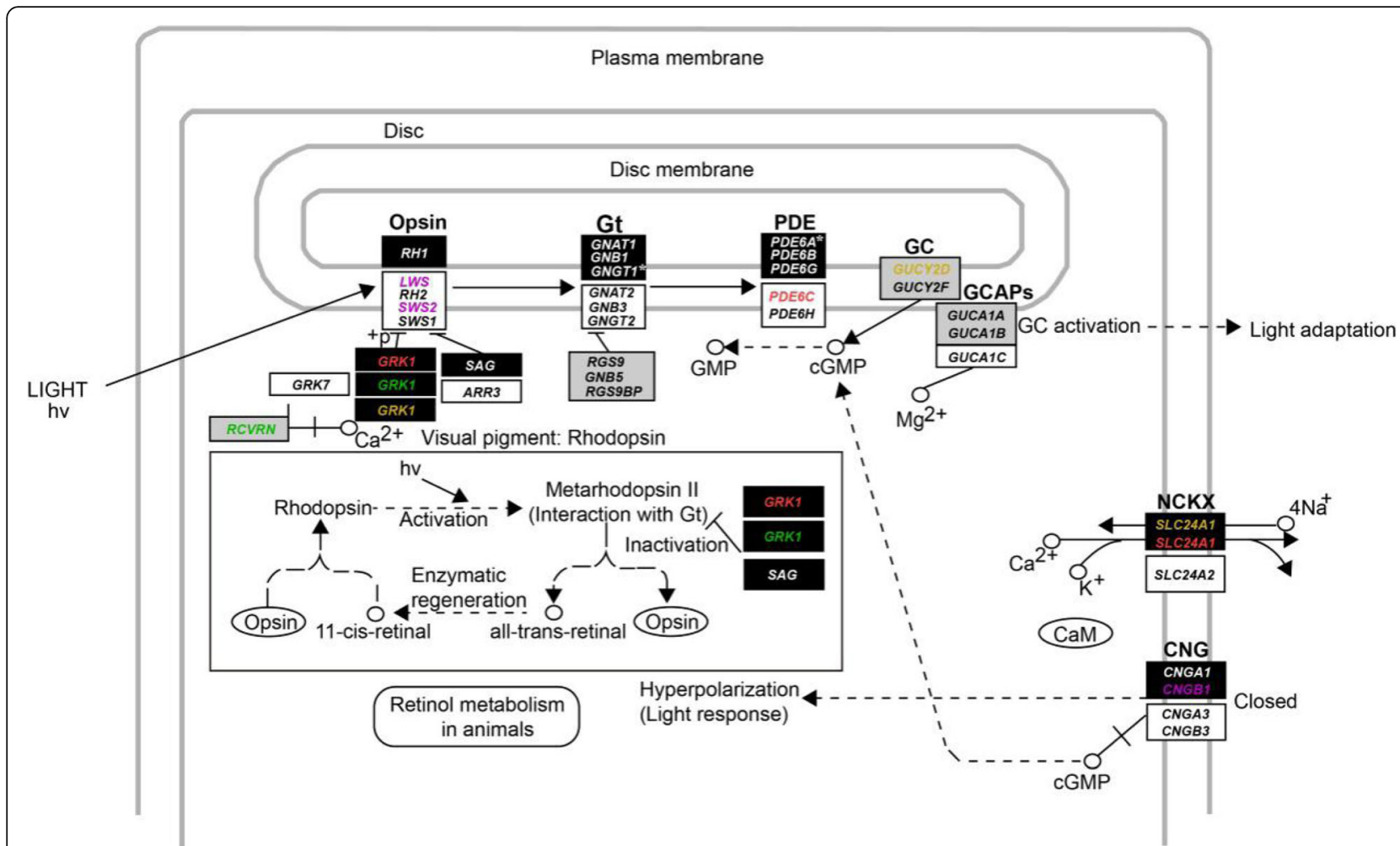

Fig. 2 Positively selected genes mapped onto the phototransduction pathway. Only the positively selected genes found in the common ancestor of extant birds (red), ancestral Carinatae (green), falcons (orange) and owls (violet) are shown. For convenience, both the genes involved in the rod phototransduction pathway (according to KEGG pathway map 04744) and the cone phototransduction pathway are shown. The genes that are involved in the phototransduction pathway of rods, cones and both are shown as dark rectangles, white rectangles and gray rectangles, respectively [12, 13]. *Represents two lost rod-expressed genes, GNGT1 and PDE6A, in both reptiles and birds based on previous studies [7, 9]

different bird lineages to their specific light environments [1].

\section{Conclusions}

Nocturnal activity in living birds, which widely occurs in nocturnal and many diurnal avian lineages, has long been documented. In this study, we reconstructed the evolutionary history of the diel activity patterns within birds using a molecular approach. Our results show that the common ancestor of living birds and ancestral Carinatae was considerably active in dim-light conditions, suggesting a deep evolutionary origin of nocturnality in birds. Further analyses indicated that the diel activity pattern of birds may have remained relatively unchanged with the subsequent diversification of most bird lineages, suggesting that the widespread nocturnality of living birds was more likely retained from their common ancestors than independently derived. The phylogenetic analyses of phototransduction genes show that functional enhancement of the two rod-expressed genes GRK1 and SLC24A1 underlies the widespread nocturnality of living birds. Moreover, photoresponse recovery genes were found to be frequently subjected to positive selection in diverse bird lineages, suggesting that birds have widely evolved an enhanced ability to detect motion.

\section{Methods}

\section{Taxa and sequences used}

The retinal transcriptome data of 17 bird species published in our previous studies [7, 8], along with data from all of the other reptiles and birds with genomes available in GenBank, were used in this study (Additional file 1). In total, 95 species were used, including 80 bird species from 34 orders, representing the majority of living bird orders (34/39) [32]. For the 95 species used, the coding sequences of 33 phototransduction genes were obtained. We used the online software webPRANK (http:// www.ebi.ac.uk/goldman-srv/webprank/) for sequence alignment, and individual sequences with low identities, long indels, multiple ambiguous bases $(\mathrm{Ns})$, and/or too short a length were removed or replaced with other relevant transcript variants. After this pruning, high-quality alignments were constructed, and their translated protein sequences were blasted against the nonredundant protein sequence database to confirm that the sequences were trimmed and/or inferred correctly. 
Table 1 Positively selected genes detected based on the PAML branch-site model. For convenience, only the $\omega$ values of foreground branches are shown. Only the positively selected sites with a high posterior probability support (>0.900) are shown

\begin{tabular}{|c|c|c|c|c|c|c|}
\hline Branches/Genes & Parameter estimates & $2 \Delta \mathrm{L}$ & df & $P$-value & $\begin{array}{l}\text { Corrected } \\
\text { P-value }\end{array}$ & Positively selected sites \\
\hline \multicolumn{7}{|l|}{ (A) } \\
\hline \multirow[t]{2}{*}{ GRK1 } & $p_{0}=0.929 p_{1}=0.051 p_{2 a}=0.017 p_{2 b}=0.000$ & 22.82 & 1 & $1.778 \mathrm{E}-06$ & $2.489 \mathrm{E}-05$ & $182 \mathrm{~S}, 269 \mathrm{E}$ \\
\hline & $\omega_{0}=0.038 \omega_{1}=1.000 \omega_{2 a}=999.000 \omega_{2 b}=999.000$ & & & & & \\
\hline \multirow[t]{2}{*}{$R C V R N$} & $p_{0}=0.915 p_{1}=0.022 p_{2 a}=0.060 p_{2 b}=0.001$ & 4.46 & 1 & 0.034 & 0.612 & $5 \mathrm{~K}, 19 \mathrm{~T}, 37 \mathrm{R}$ \\
\hline & $\omega_{0}=0.019 \omega_{1}=1.000 \omega_{2 a}=28.660 \omega_{2 b}=28.660$ & & & & & \\
\hline \multicolumn{7}{|l|}{ (B) } \\
\hline \multirow[t]{2}{*}{ GRK1 } & $p_{0}=0.902 p_{1}=0.050 p_{2 a}=0.044 p_{2 b}=0.002$ & 5.71 & 1 & 0.016 & 0.224 & $9 \mathrm{~K}, 25 \mathrm{~K}, 252 \mathrm{~N}, 316 \mathrm{I}$ \\
\hline & $\omega_{0}=0.038 \omega_{1}=1.000 \omega_{2 a}=11.732 \omega_{2 b}=11.732$ & & & & & \\
\hline \multicolumn{7}{|l|}{ (C) } \\
\hline \multirow[t]{2}{*}{ LWS } & $p_{0}=0.898 p_{1}=0.091 p_{2 a}=0.008 p_{2 b}=0.000$ & 7.09 & 1 & 0.007 & 0.112 & $70 S, 254 S$ \\
\hline & $\omega_{0}=0.036 \omega_{1}=1.000 \omega_{2 a}=40.133 \omega_{2 b}=40.133$ & & & & & \\
\hline \multicolumn{7}{|l|}{ (D) } \\
\hline \multirow[t]{2}{*}{ SWS1 } & $p_{0}=0.948 p_{1}=0.010 p_{2 a}=0.040 p_{2 b}=0.000$ & 4.55 & 1 & 0.032 & 0.512 & $78 \mathrm{~T}$ \\
\hline & $\omega_{0}=0.023 \omega_{1}=1.000 \omega_{2 a}=10.955 \omega_{2 b}=10.955$ & & & & & \\
\hline \multicolumn{7}{|l|}{ (E) } \\
\hline \multirow[t]{2}{*}{ GUCY2D } & $p_{0}=0.906 p_{1}=0.089 p_{2 a}=0.003 p_{2 b}=0.000$ & 6.03 & 1 & 0.014 & 0.126 & $459 F, 565 K$ \\
\hline & $\omega_{0}=0.046 \omega_{1}=1.000 \omega_{2 a}=302.150 \omega_{2 b}=302.150$ & & & & & \\
\hline \multicolumn{7}{|l|}{$(\mathrm{F})$} \\
\hline \multirow[t]{2}{*}{ GUCY2D } & $p_{0}=0.905 p_{1}=0.089 p_{2 a}=0.004 p_{2 b}=0.000$ & 5.68 & 1 & 0.017 & 0.153 & $69 \mathrm{~K}, 161 \mathrm{D}$ \\
\hline & $\omega_{0}=0.046 \omega_{1}=1.000 \omega_{2 a}=50.303 \omega_{2 b}=50.303$ & & & & & \\
\hline \multicolumn{7}{|l|}{ (G) } \\
\hline \multirow[t]{2}{*}{ CNGB1 } & $p_{0}=0.896 p_{1}=0.087 p_{2 a}=0.014 p_{2 b}=0.001$ & 3.83 & 1 & 0.050 & 1.150 & $346 \mathrm{~N}, 475 \mathrm{G}$ \\
\hline & $\omega_{0}=0.047 \omega_{1}=1.000 \omega_{2 a}=6.517 \omega_{2 b}=6.517$ & & & & & \\
\hline \multirow[t]{2}{*}{ LWS } & $p_{0}=0.877 p_{1}=0.090 p_{2 a}=0.028 p_{2 b}=0.002$ & 16.28 & 1 & 5.439E-05 & $8.702 \mathrm{E}-04$ & $126 \mathrm{~K}, 130 \mathrm{R}, 237 \mathrm{H}$ \\
\hline & $\omega_{0}=0.036 \omega_{1}=1.000 \omega_{2 a}=67.591 \omega_{2 b}=67.591$ & & & & & \\
\hline \multirow[t]{2}{*}{ SWS2 } & $p_{0}=0.824 p_{1}=0.125 p_{2 a}=0.042 p_{2 b}=0.006$ & 10.94 & 1 & 0.000 & 0.013 & $18 \mathrm{~A}, 42 \mathrm{~K}, 43 \mathrm{~A}$ \\
\hline & $\omega_{0}=0.038 \omega_{1}=1.000 \omega_{2 a}=237.344 \omega_{2 b}=237.344$ & & & & & \\
\hline \multicolumn{7}{|l|}{$(\mathrm{H})$} \\
\hline \multirow[t]{2}{*}{ GRK1 } & $p_{0}=0.878 p_{1}=0.047 p_{2 a}=0.070 p_{2 b}=0.003$ & 13.14 & 1 & 0.000 & 0.004 & $\begin{array}{l}2 \mathrm{~L}, 9 \mathrm{~K}, 14 \mathrm{E}, 16 \mathrm{C} \\
25 \mathrm{~K}, 29 \mathrm{~N}, 33 \mathrm{~N}, 44 \mathrm{E}\end{array}$ \\
\hline & $\omega_{0}=0.037 \omega_{1}=1.000 \omega_{2 a}=15.634 \omega_{2 b}=15.634$ & & & & & $\begin{array}{l}197 \mathrm{E}, 250 \mathrm{R}, 289 \mathrm{~A}, \\
292 \mathrm{~T}, 303 \mathrm{~K}, 306 \mathrm{Y}\end{array}$ \\
\hline \multirow[t]{2}{*}{ GUCY2D } & $p_{0}=0.903 p_{1}=0.088 p_{2 a}=0.006 p_{2 b}=0.000$ & 8.35 & 1 & 0.003 & 0.027 & $175 \mathrm{G}$ \\
\hline & $\omega_{0}=0.046 \omega_{1}=1.000 \omega_{2 a}=999.000 \omega_{2 b}=999.000$ & & & & & \\
\hline \multirow[t]{2}{*}{ SLC24A1 } & $p_{0}=0.736 p_{1}=0.257 p_{2 a}=0.003 p_{2 b}=0.001$ & 4.93 & 1 & 0.026 & 0.520 & 2331 \\
\hline & $\omega_{0}=0.048 \omega_{1}=1.000 \omega_{2 a}=42.679 \omega_{2 b}=42.679$ & & & & & \\
\hline \multicolumn{7}{|l|}{ (l) } \\
\hline \multirow[t]{2}{*}{ LWS } & $p_{0}=0.902 p_{1}=0.092 p_{2 a}=0.004 p_{2 b}=0.000$ & 5.00 & 1 & 0.025 & 0.400 & $97 \mathrm{~S}$ \\
\hline & $\omega_{0}=0.037 \omega_{1}=1.000 \omega_{2 a}=58.101 \omega_{2 b}=58.101$ & & & & & \\
\hline \multicolumn{7}{|c|}{ 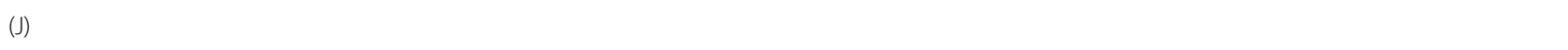 } \\
\hline \multirow[t]{2}{*}{ GUCY2F } & $p_{0}=0.770 p_{1}=0.228 p_{2 a}=0.001 p_{2 b}=0.000$ & 8.15 & 1 & 0.004 & 0.100 & $306 \mathrm{E}$ \\
\hline & $\omega_{0}=0.063 \omega_{1}=1.000 \omega_{2 a}=244.082 \omega_{2 b}=244.082$ & & & & & \\
\hline LWS & $p_{0}=0.908 p_{1}=0.084 p_{2 a}=0.006 p_{2 b}=0.000$ & 4.01 & 1 & 0.045 & 0.720 & $43 \mathrm{~K}$ \\
\hline
\end{tabular}


Table 1 Positively selected genes detected based on the PAML branch-site model. For convenience, only the $\omega$ values of foreground branches are shown. Only the positively selected sites with a high posterior probability support (>0.900) are shown (Continued)

\begin{tabular}{|c|c|c|c|c|c|c|}
\hline Branches/Genes & Parameter estimates & $2 \Delta \mathrm{L}$ & $d f$ & $P$-value & $\begin{array}{l}\text { Corrected } \\
P \text {-value }\end{array}$ & Positively selected sites \\
\hline & $\omega_{0}=0.038 \omega_{1}=1.000 \omega_{2 a}=59.283 \omega_{2 b}=59.283$ & & & & & \\
\hline \multicolumn{7}{|l|}{$(\mathrm{K})$} \\
\hline \multirow[t]{2}{*}{ LWS } & $p_{0}=0.904 p_{1}=0.082 p_{2 a}=0.011 p_{2 b}=0.001$ & 6.41 & 1 & 0.011 & 0.176 & $42 \mathrm{~A}, 88 \mathrm{~L}$ \\
\hline & $\omega_{0}=0.038 \omega_{1}=1.000 \omega_{2 a}=213.404 \omega_{2 b}=213.404$ & & & & & \\
\hline \multirow[t]{2}{*}{ RCVRN } & $p_{0}=0.960 p_{1}=0.023 p_{2 a}=0.015 p_{2 b}=0.000$ & 7.22 & 1 & 0.007 & 0.126 & \\
\hline & $\omega_{0}=0.019 \omega_{1}=1.000 \omega_{2 a}=999.000 \omega_{2 b}=999.000$ & & & & & \\
\hline
\end{tabular}

Corrected $P$-value, Bonferroni multiple testing correction, $P$ values are corrected by multiplying them by the number of branches tested of each gene. Significance level is $P<0.05$

\section{Positive selection analyses}

We analyzed our focal genes for evidence of positive selection using the branch model, the branch-site model and clade model $\mathrm{C}$ implemented in the Codeml program of PAML [26]. Thus, a codon-based maximum likelihood method was used to estimate the ratio of nonsynonymous to synonymous substitutions per site $(\mathrm{dN} / \mathrm{dS}$ or $\omega$ ), and likelihood ratio tests (LRTs) were employed to determine whether the results were statistically significant. A statistically significant value of $\omega>1$ suggested positive selection. $P$ values were subjected to Bonferroni correction. Upon analysis, we constructed an unrooted species tree following published studies [14-25] and the Tree of Life Web Project (http://tolweb.org/Passerifor mes). The phylogenetic relationships among bird orders followed one genome-level study [18].

Table 2 Positively selected genes of the entire clade of birds based on the clade model $\mathrm{C}$

\begin{tabular}{lllll}
\hline Genes & Parameter estimates & $2 \Delta \mathrm{L}$ & $\mathrm{df}$ & P-value \\
\hline CNGA1 & $p_{0}=0.903 p_{1}=0.086 p_{2}=0.010$ & 17.88 & 1 & $2.350 \mathrm{E}-05$ \\
& $\omega_{0}=0.041 \omega_{1}=1.000 \omega_{2}=2.231$ & & & \\
CNGB3 & $p_{0}=0.808 p_{1}=0.187 p_{2}=0.004$ & 9.80 & 1 & 0.001 \\
& $\omega_{0}=0.052 \omega_{1}=1.000 \omega_{2}=5.094$ & & & \\
PDE6B & $p_{0}=0.933 p_{1}=0.063 p_{2}=0.002$ & 4.56 & 1 & 0.032 \\
& $\omega_{0}=0.036 \omega_{1}=1.000 \omega_{2}=2.620$ & & & \\
PDE6C & $p_{0}=0.851 p_{1}=0.136 p_{2}=0.011$ & 38.37 & 1 & $5.845 \mathrm{E}-10$ \\
& $\omega_{0}=0.048 \omega_{1}=1.000 \omega_{2}=3.145$ & & & \\
SAG & $p_{0}=0.806 p_{1}=0.177 p_{2}=0.015$ & 24.06 & 1 & $9.336 \mathrm{E}-07$ \\
& $\omega_{0}=0.077 \omega_{1}=1.000 \omega_{2}=2.621$ & & & \\
SLC24A1 & $p_{0}=0.737 p_{1}=0.249 p_{2}=0.013$ & 19.75 & 1 & $8.816 \mathrm{E}-06$ \\
& $\omega_{0}=0.050 \omega_{1}=1.000 \omega_{2}=4.065$ & & & \\
SLC24A2 & $p_{0}=0.782 p_{1}=0.193 p_{2}=0.024$ & 13.68 & 1 & 0.000 \\
& $\omega_{0}=0.051 \omega_{1}=1.000 \omega_{2}=2.917$ & & & \\
\hline
\end{tabular}

$2 \Delta \mathrm{L}$ : twice the difference of likelihood values between two nested models; df: degrees of freedom; $p$ : proportion of sites in different site classes $\left(p_{0}, p_{1}\right.$ and $\left.p_{2}\right)$

\section{Branch model}

We used a two-rate branch model to detect possible positive selection signals along the branches of interest. For the analyses, each of our focal branches was designated as the foreground branch, and the others were treated as background branches. In the two-rate branch model, foreground branches and background branches were assumed to have different $\omega$ values, and the goodness of fit of the two-rate branch model was analyzed using an LRT. Using an LRT, the two-rate branch model was compared with the one-rate branch model that assumed a single $\omega$ value across the tree, and if a statistically significant value of $\omega>1$ was detected in a foreground branch, the two-rate branch model was further compared with a two-rate branch model in which $\omega$ was constrained on all branches to $\omega=1$ to further assess whether the $\omega>1$ of the foreground branch was statistically supported.

\section{Branch-site model}

We used a branch-site model (Test 2) to look for evidence of positively selected sites on our focal branches. For the analyses, four classes of sites were assumed. Site class $0 \quad(0<\omega 0<1)$ and site class $1(\omega 1=1)$ were assumed to represent evolutionarily conserved and neutral codons, respectively, for both background branches and foreground branches. Site classes $2 \mathrm{a}$ and $2 \mathrm{~b}$ were assumed to represent evolutionarily conserved $(0<\omega 0<1)$ and neutral $(\omega 1=1)$ codons, respectively, for background branches but were allowed to be under positive selection $(\omega 2>1)$ on the foreground branches. In the branch-site model, a modified model A was compared with its corresponding null model with $\omega=1$ constrained to determine the statistical significance. The empirical Bayes method was used to detect positively selected sites.

\section{Clade model C}

We used clade model $\mathrm{C}$ to examine evidence for possible divergent selection on phototransduction genes in birds. To this end, the entire bird clade was designated as the 
foreground clade, while the others were treated as the background clades. Model $\mathrm{C}$ assumed three site classes, where site class $0(0<\omega 0<1)$, site class $1(\omega 1=1)$ and site class $2(\omega 2,3)$ represented evolutionarily conserved, evolutionarily neutral and potentially positively selected codons, respectively, in both the background and foreground clades. Clade model $\mathrm{C}$ compares model $\mathrm{C}$ with its corresponding null model M2a_rel to determine LRT statistical significance.

\section{Robustness test of positively selected genes}

To test the robustness of our results, we examined the dependency of our positively selected genes on the initial values of kappa and omega. Thus, two different initial values of kappa (kappa $=0.5,3.0)$ and omega $(\omega=0.5$, 2.0) were used for the positive selection analyses, and four independent runs were conducted for each of the positively selected genes. In addition, we used BUSTED, implemented in HyPhy software (version 2.2.4) [33, 34], to confirm the positively selected genes identified by the branch-site model of PAML. BUSTED differs from PAML in that it allows for the occurrence of positive selection on both foreground and background branches (unconstrained model), while PAML allows positive selection only on foreground branches. The fit of the unconstrained model was compared with that of the null model that disallowed $\omega>1$ among the foreground branches. Statistical significance was determined by an LRT.

\section{Supplementary information}

Supplementary information accompanies this paper at https://doi.org/10. 1186/s12862-019-1508-y.

Additional file 1. Species and sequences used in this study.

Additional file 2. Positive selection analyses using BUSTED.

\section{Abbreviations}

LRT: Likelihood ratio tests; MPE: Molecular phyloecological

\section{Acknowledgements}

Not applicable.

\section{Author's contributions}

YW conceived of the study, performed analyses, and wrote the manuscript. The author read and approved the final manuscript.

\section{Funding}

This research was supported by the That the National Natural Science Foundation of China (grant number, 31770401) represents an institution and hence no dot should be there.

Foundation of China (grant number, 31770401) and the Fundamental

Research Funds for the Central Universities.

\section{Availability of data and materials}

All data needed to evaluate the conclusions in the paper are present in the paper and/or the additional files.

Ethics approval and consent to participate Not applicable.
Consent for publication

Not applicable.

\section{Competing interests}

The author declares that he has no competing interests.

Received: 8 May 2019 Accepted: 5 September 2019

Published online: 16 October 2019

\section{References}

1. Maitin G. Birds by night. London: T \& A D Poyser; 1990

2. Martin GR. The sensory ecology of birds. Oxford: Oxford University Press; 2017.

3. La VT. Diurnal and nocturnal birds vocalize at night: a review. Condor. 2012; 114:245-57.

4. Roots C. Nocturnal animals. London: Greenwood Press; 2006.

5. Duc DL, Schöneberg T. Adaptation to nocturnality - learning from avian genomes. BioEssays. 2016;38:694-703.

6. Mouritsen H, Feenders G, Liedvogel M, Wada K, Jarvis ED. Night-vision brain area in migratory songbirds. Proc Natl Acad Sci U S A. 2004;102:8339-44.

7. Wu Y, Hadly EA, Teng W, Hao Y, Liang W, Liu Y, Wang H. Retinal transcriptome sequencing sheds light on the adaptation to nocturnal and diurnal lifestyles in raptors. Sci Rep. 2016;6:33578.

8. Wu $\mathrm{Y}, \mathrm{Wang} \mathrm{H}$. Convergent evolution of bird-mammal shared characteristics for adapting to nocturnality. Proc R Soc B. 2019;286:20182185.

9. Wu Y, Wang H, Hadly EA. Invasion of ancestral mammals into dim-light environments inferred from adaptive evolution of the phototransduction genes. Sci Rep. 2017;7:46542.

10. Wu $Y$, Wang $H$, Wang $H$, Feng J. Arms race of temporal partitioning between carnivorous and herbivorous mammals. Sci Rep. 2018:8:1713.

11. Wu $\mathrm{Y}$, Wang $H$, Wang $H$, Hadly EA. Rethinking the origin of primates by reconstructing their diel activity patterns using genetics and morphology. Sci Rep. 2017;7:11837.

12. Lamb TD. Evolution of phototransduction, vertebrate photoreceptors and retina. Prog Retin Eye Res. 2013;36:52-119.

13. Larhammar D, Nordström K, Larsson TA. Evolution of vertebrate rod and cone phototransduction genes. Philos Trans R Soc B. 2009;364:2867-80.

14. Crawford NG, Faircloth BC, McCormack JE, Brumfield RT, Winker K, Glenn TC. More than 1000 ultraconserved elements provide evidence that turtles are the sister group of archosaurs. Biol Lett. 2012;8:783-6.

15. Crawford NG, Parham JF, Sellas AB, Faircloth BC, Glenn TC, Papenfuss TJ, Henderson JB, Hansen MH, Simison WB. A phylogenomic analysis of turtles. Mol Phylogenet Evol. 2015;83:250-7.

16. Fuchs J, Johnson JA, Mindell DP. Rapid diversification of falcons (Aves: Falconidae) due to expansion of open habitats in the Late Miocene. Mol Phylogenet Evol. 2015;82:166-82.

17. Green R, Braun E, Armstrong J, Earl D, Nguyen N, Hickey G, Vandewege M, St John J, Capella-Gutiérrez S, Castoe T, et al. Three crocodilian genomes reveal ancestral patterns of evolution among archosaurs. Science. 2014;346:1254449.

18. Jarvis E, Mirarab S, Aberer A, Li B, Houde P, Li C, Ho S, Faircloth B, Nabholz $B$, Howard J, et al. Whole-genome analyses resolve early branches in the tree of life of modern birds. Science. 2014;346:1320-31.

19. Jønsson KA, Fjeldså J. A phylogenetic supertree of oscine passerine birds (Aves: Passeri). Zool Scr. 2006:35:149-86.

20. Kriegs JO, Matzke A, Churakov G, Kuritzin A, Mayr G, Brosius J, Schmitz J. Waves of genomic hitchhikers shed light on the evolution of gamebirds (Aves: Galliformes). BMC Evol Biol. 2007;7:190.

21. Le Duc D, Renaud G, Krishnan A, Almén MS, Huynen L, Prohaska SJ, Ongyerth $M$, Bitarello BD, Schiöth $H B$, Hofreiter $M$, et al. Kiwi genome provides insights into evolution of a nocturnal lifestyle. Genome Biol. 2015;16:147.

22. Lerner HR, Mindell DP. Phylogeny of eagles, Old World vultures, and other Accipitridae based on nuclear and mitochondrial DNA. Mol Phylogenet Evol. 2005;37:327-46

23. Pyron RA, Burbrink FT, Wiens JJ. A phylogeny and revised classification of Squamata, including 4161 species of lizards and snakes. BMC Evol Biol. 2013:13:93

24. Wink M, El-Sayed A-A, Sauer-Gürth H, Gonzalez J. Molecular phylogeny of owls (Strigiformes) inferred from DNA sequences of the mitochondrial cytochrome b and the nuclear RAG-1 gene. Ardea. 2009;97:581-91. 
25. Wright TF, Schirtzinger EE, Matsumoto T, Eberhard JR, Graves GR, Sanchez JJ, Capelli S, Müller H, Scharpegge J, Chambers GK, et al. A multilocus molecular phylogeny of the parrots (Psittaciformes): support for a Gondwanan origin during the cretaceous. Mol Biol Evol. 2008;25:2141-56.

26. Yang Z. PAML 4: phylogenetic analysis by maximum likelihood. Mol Biol Evol. 2007;24:1586-91.

27. Umino Y, Herrmann R, Chen C, Barlow R, Arshavsky V, Solessio E. The relationship between slow photoresponse recovery rate and temporal resolution of vision. J Neurosci. 2012;32:14364-73.

28. DeCandido R, Allen D. Nocturnal hunting by peregrine falcons at the empire state building, New York City. Wilson J Ornithol. 2006;118:53-8,

29. del Hoyo J, Elliot A, Sargatal J. Handbook of birds of the world. In: New world vultures to guineafowl, vol. 2. Barcelona: Lynx Edicions; 1994.

30. Hirata K, Nakahama S, Yoshioka T. Hunting in the dark by a peregrine falcon (Falco peregrinus). Slovak Raptor J. 2014:7:85-7.

31. Negro J, Bustamante J, Melguizo C, Ruiz J, Grande J. Nocturnal activity of lesser kestrels under artificial lighting conditions in Seville, Spain. J Raptor Res. 2000;34:327-9.

32. Gill F, Donsker D. IOC world bird list (v8.1); 2018. https://doi.org/10.14344/ IOC.ML.8.1.

33. Kosakovsky Pond SL, Frost S, Muse S. HyPhy: hypothesis testing using phylogenies. Bioinformatics. 2014;21:676-9.

34. Murrell B, Weaver S, Smith MD, Wertheim JO, Murrell S, Aylward A, Eren K, Pollner T, Martin DP, Smith DM, et al. Gene-wide identification of episodic selection. Mol Biol Evol. 2015;32:1365-71.

\section{Publisher's Note}

Springer Nature remains neutral with regard to jurisdictional claims in published maps and institutional affiliations.

Ready to submit your research? Choose BMC and benefit from:

- fast, convenient online submission

- thorough peer review by experienced researchers in your field

- rapid publication on acceptance

- support for research data, including large and complex data types

- gold Open Access which fosters wider collaboration and increased citations

- maximum visibility for your research: over $100 \mathrm{M}$ website views per year

At $\mathrm{BMC}$, research is always in progress.

Learn more biomedcentral.com/submissions 